\title{
Riqueza de vespas sociais (Hymenoptera: Vespidae) de Cerrado no sudeste do Brasil
}

As vespas sociais pertencem à ordem Hymenoptera, família Vespidae, subfamílias Stenogastinae, Vespinae e Polistinae, sendo a única que ocorre em território brasileiro. Estudos sobre a diversidade de vespas sociais no estado de Minas Gerais vem apresentando um crescente número nos últimos anos, porém se tratando do bioma Cerrado, que compreende mais da metade do território do estado, os estudos são escassos. Vespas sociais apresentam uma grande importância ecológica, pois participam do equilíbrio trófico dos ecossistemas, atuando como polinizadores e predadores de insetos. O presente trabalho tem como objetivo realizar o primeiro inventário da riqueza de vespas sociais (Hymenoptera, Vespidae, Polistinae) do Parque Nacional da Serra da Canastra (PNSC) no município de São Roque de Minas, Minas Gerais, Brasil. Além disso, avaliar a riqueza e distribuição de espécies destes insetos em diferentes fitofisionomias dentro do mesmo Parque. As coletas foram realizadas no ano de 2018 , nos meses de fevereiro, abril, setembro e novembro, através de duas metodologias: armadilhas atrativas e busca ativa. Coletou-se um total de 2239 espécimes de vespas sociais de 33 espécies e nove gêneros, obtendo a maior riqueza para estudo realizados em áreas de Cerrado. Além disso, fica claro que a similaridade das fitofisionomias é mais relevante na composição de espécies que a distâncias entre as áreas de estudo.

\section{Richness of social wasp (Hymenoptera: Vespidae) in the Brazilian southern Cerrado}

\begin{abstract}
The social wasps belong to the order Hymenoptera, family Vespidae, subfamilies Stenogastinae, Vespinae and Polistinae, being the only one that occurs in Brazilian territory. Studies on the diversity of social wasps in the state of Minas Gerais have been showing an increasing number in recent years, however in the case of the Cerrado biome, which comprises more than half of the state's territory, studies are scarce. Social wasps are of great ecological importance, as they participate in the trophic balance of ecosystems, acting as pollinators and insect predators. This work aims to carry out the first inventory of the wealth of social wasps (Hymenoptera, Vespidae, Polistinae) from the Serra da Canastra National Park (PNSC) in the municipality of São Roque de Minas, Minas Gerais, Brazil. In addition, to evaluate the richness and species distribution of these insects in different phytophysiognomies within the same Park. The collections were carried out in 2018 , in the months of February, April, September and November, using two methodologies: attractive traps and active search. A total of 2239 specimens of social wasps of 33 species and nine genera were collected, obtaining the greatest wealth for studies carried out in Cerrado areas. In addition, it is clear that the similarity of phytophysiognomies is more relevant in the composition of species than the distances between the study areas.
\end{abstract}

Keywords: Conservation; Inventory; Polistinae; Serra da Canastra.

Topic: Conservação da Biodiversidade

Reviewed anonymously in the process of blind peer.
Received: $11 / 09 / 2020$

Approved: 01/11/2020
Lucas de Oliveira Vicente (iD

Instituto Federal de Minas Gerais, Brasil

http://lattes.cnpq.br/3317814198416608

http://orcid.org/0000-0002-0284-910X

lucas.vicentte@yahoo.com.br

Gabriel de Castro Jacques

Instituto Federal de Minas Gerais, Brasil

http://lattes.cnpq.br/3848049450080555

http://orcid.org/0000-0002-9619-6065

gabriel.jacques@ifmg.edu.br

Marcos Magalhães de Souza (iD)

Instituto Federal do Sul de Minas, Brasil

http://lattes.cnpq.br/2334845279402555

http://orcid.org/0000-0003-0415-1714

marcos.souza@ifsuldeminas.edu.br
Bruno Senna Corrêa (iD)

Centro Federal de Educação Tecnológica de Minas Gerais, Brasil

http://lattes.cnpq.br/4682930954371672

http://orcid.org/0000-0002-3554-9234

bruno.senna@gmail.com
Referencing this:

VICENTE, L. O.; JACQUES, G. C.; SOUZA, M. M.; CORRÊA, B. S.. Riqueza de vespas sociais (Hymenoptera: Vespidae) de Cerrado no sudeste do Brasil. Nature and Conservation, v.13, n.4, p.1-11, 2020. DOI: http://doi.org/10.6008/CBPC2318-2881.2020.004.0001 


\section{INTRODUÇÃO}

As vespas sociais pertencem à ordem Hymenoptera e família Vespidae com seis subfamílias Stenogastinae, Vespinae e Polistinae (ANDENA et al., 2009). A subfamília Polistinae é a única que ocorre em território brasileiro com 21 gêneros e 344 espécies (HERMES et al., 2020).

$\mathrm{O}$ crescente interesse em estudos de diversidade de vespas sociais no Brasil se deve à reconhecida importância ecológica que esses insetos apresentam atuando no equilíbrio trófico dos ecossistemas (PREZOTO et al., 2008; PREZOTO et al., 2019) como predadores de insetos (PREZOTO et al., 1999; JACQUES et al., 2019) e polinizadores (HERMES et al., 2006; CLEMENTE et al., 2009). Além disso, podem ser usados como bioindicadores do estado de conservação de florestas ripárias (SOUZA et al., 2010), o que é relevante, considerando que a fragmentação florestal afeta negativamente suas populações (GRAÇA et al., 2018; BUENO et al., 2019).

Apesar dessa relevância e do crescente esforço de estudos de inventários de espécies (BARBOSA et a., 2016; SOUZA et al., 2017), há uma concentração desses trabalhos em áreas de floresta Atlântica (SOUZA et al., 2020a), sendo necessário atenção às áreas de Cerrado, que compreende $57 \%$ do território mineiro (IBGE, 2004), contudo, se registra poucos estudos (ELPINO-CAMPOS et al., 2007; SIMÕES et al., 2012, JACQUES et al., 2015; 2018), com quase nenhuma informação para Unidades de Conservação (SOUZA et al., 2020b).

O Cerrado é um dos hotspots mundiais de biodiversidade, apresentando uma rica diversidade de espécies endêmicas (MYERS et al., 2000; SILVA et al., 2002). A cobertura original do cerrado brasileiro já foi reduzida em mais de 55\% (MACHADO et al., 2004a), comprometendo muito a sua biodiversidade. Esse fato, somado ao alto endemismo (FELFILI et al., 1997; RATTER et al., 2003) e aos pequenos percentuais, cerca de 2,2\% apenas, de áreas legalmente declaradas como áreas de conservação (KINK et al., 2005), indicam os riscos de perda das informações sobre a biodiversidade da região (FELFILI et al., 2001).

O Parque Nacional da Serra da Canastra (PNSC) inclui-se nos domínios do bioma Cerrado, com influência do bioma Mata Atlântica, sendo uma área cuja prioridade de conservação é extremamente alta (DRUMONND et al., 2005). Considerando que estudos de inventários são essenciais para se conhecer os recursos naturais disponíveis em determinada área, bem como contribui para compreensão das características ecológicas de um ecossistema e seu manejo, é justificável estudos dessa natureza, sobretudo em áreas de proteção ambiental.

Considerando essas informações, o presente trabalho tem como objetivo realizar o inventário da riqueza de vespas sociais (Hymenoptera, Vespidae, Polistinae) do Parque Nacional da Serra da Canastra (PNSC) no município de São Roque de Minas, Minas Gerais, Brasil. Além disso, avaliar a riqueza e distribuição de espécies destes insetos em diferentes fitofisionomias dentro do mesmo Parque.

\section{MATERIAIS E MÉTODOS}

O estudo foi realizado no Parque Nacional da Serra da Canastra (20 18' 16" S e 46 $35^{\prime}$ 56" O), no 
município de São Roque de Minas, Minas Gerais, Brasil. A amostragem foi realizada em três áreas dentro da Unidade de Conservação (Figura 1), selecionadas pela presença de cursos de água e pela facilidade da logística de acesso.

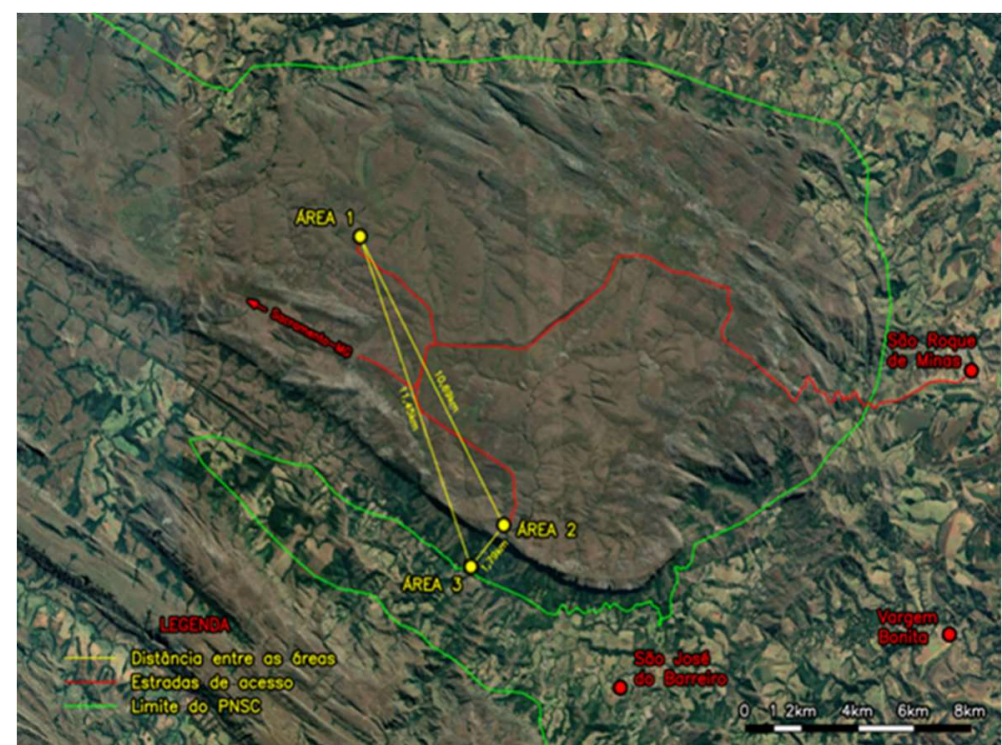

Figura 1: Imagem de satélite obtida através do programa Google Earth Pro ${ }^{\oplus}$, com a localização das três áreas de estudo, onde foram coletadas vespas sociais no Parque Nacional da Serra da Canastra, município de São Roque de Minas, Minas Gerais, Brasil.

A primeira área de estudo localiza-se na estrada de acesso a Cachoeira do Rolinho, em uma região formada por Cerrado stricto sensu. Nessa área a paisagem é composta por um estrato herbáceo, dominado principalmente por gramíneas (Poaceae), e um estrato de árvores e arbustos tortuosos, com ramificações irregulares e retorcidas, característico do bioma Cerrado (EITEN, 1994). Essa área foi denominada de 'Área 1 - Cachoeira do Rolinho'. A segunda área de estudo, a 10,69 km da primeira área, localiza-se na parte alta da Cachoeira Casca d'Anta, denominada como 'Área 2 - Cachoeira Casca d'Anta'. Essa área se assemelha muito com a 'Área 1 - Cachoeira do Rolinho', em termos de fitofisionomia.

Já a terceira área de estudo, a 1,79 km da segunda área, localiza-se na parte baixa do PNSC. A fitofisionomia é a Floresta Estacional Semidecidual, vegetação pertencente ao bioma Mata Atlântica, com transição para área de Cerrado. Nessa área fica notório a mudança de fitofisionomia, sendo possível observar a formação de uma mata mais fechada, com um dossel mais volumoso e a presença da camada de serapilheira no solo. Além disso, é uma área sob influência antrópica como quiosques, banheiros, postes de iluminação e trilhas. Essa área encontra-se logo acima da margem esquerda do caminho de acesso à parte baixa da Cachoeira Casca d'Anta, ficando denominada como 'Área 03 - Parte Baixa'.

A amostragem das vespas sociais foi realizada em quatro campanhas ao longo do ano de 2018 , nos meses de fevereiro, abril, setembro e novembro. As campanhas tinham duração de uma semana e as coletas foram através de duas metodologias: armadilhas atrativas e busca ativa. As armadilhas atrativas foram elaboradas com garrafas do tipo 'PET' (Polietileno Tereftalato) de dois litros com três aberturas triangulares laterais $(2 \times 2 \times 2 \mathrm{~cm})$ à distância de aproximadamente $10 \mathrm{~cm}$ da base (SOUZA et al., 2006; SOUZA et al., 2015), sendo instaladas à $1,5 \mathrm{~m}$ de altura do solo, e em cada armadilha foram adicionados $150 \mathrm{~mL}$ de mel 
(JACQUES et al., 2018b).

Cada área de estudo selecionada recebeu dois transectos lineares, distribuídos de forma aleatória, com o intuito de abranger uma grande parcela da área. Cada transecto linear, continha 10 armadilhas, com uma distância de 30 metros por armadilha, totalizando 300 metros em cada transecto (adaptado de MACIEL, 2017). Todas as armadilhas distribuídas nas três áreas de estudo permaneceram em campo por sete dias e foram georreferenciadas com o auxílio de um GPS de navegação GARMIN ${ }^{\circledR}$ GPSmap60csx, com o intuito de repetir a mesma distribuição em todas as campanhas.

As buscas ativas foram realizadas em dois dias por campanha, sendo oito horas por dia, totalizando 64 horas de esforço amostral por área selecionada. Essa metodologia consiste na utilização de redes entomológicas, capturando os indivíduos pousados sobre a vegetação, visitando flores ou em pleno voo (HERMES et al., 2006). Além disso, troncos, cavidades naturais (cupinzeiros abandonados e rochas), vegetação de folhas largas e construções antrópicas que ainda existem no parque foram vistoriados a procura de ninhos (SOUZA et al., 2006; ELPINO-CAMPOS et al., 2007).

As vespas sociais coletadas, tanto por armadilhas ou por busca ativa, foram preservadas em álcool 70\% (JACQUES et al., 2015) e identificadas com chaves entomológicas (RICHARDS, 1978; CARPENTER, 2004) e confirmadas pelos professores, Dr. Marcos Magalhães de Souza, Instituto Federal do Sul de Minas, Campus Inconfidentes e o Dr. Orlando Tobias da Silveira, Museu Emílio Goeldi, Belém, Pará. O material se encontra depositado no Laboratório de Entomologia do Instituto Federal de Minas Gerais - Campus Bambuí.

A diversidade e dominância de espécies, total e de cada área, foi calculada com o índice de diversidade de Shannon-Wiener $\left(H^{\prime}\right)$ e a de dominância de Berger-Parker (Dpq), através do programa Past (HAMMER et al., 2005). O total de indivíduos, a riqueza de espécies e os índices de diversidade e a de dominância de cada área foram submetidos à análise de variância pelo teste $\mathrm{F}$ e as médias comparadas pelo teste de Tukey a nível de 5\% de significância, quando aplicável, com o programa estatístico Past (HAMMER et al., 2005). Para avaliar a eficiência da coleta de cada área foi realizado curvas de acumulação de espécies, com intervalo de confiança de 95\%, usando o estimador Bootstrap 1 no software EstimateS 9.1.0 (COWELL, 2013). Este estimador utiliza informações de todas as espécies coletadas, em vez de restringir a análise de espécies raras (SANTOS, 2003).

Além disso, foi realizado também uma análise de similaridade (Cluster analysis) entre as faunas de vespas sociais coletadas nas três áreas selecionadas. Para essa análise foi utilizado o índice de similaridade de Bray-Curtis através do programa Past (HAMMER et al., 2005), que leva em consideração a ocorrência e a abundância das espécies em cada área. Foi realizada, também, outra análise de similaridade para comparar a diversidade de vespas sociais em diferentes trabalhos realizados no Cerrado em Minas Gerais (ELPINOCAMPOS et al., 2007; SIMÕES et al., 2012; JACQUES et al., 2015; 2018a). Também foi utilizado o estudo de Brunismann et al. (2016), realizado em floresta decidual, fitofisionomia considerada de domínio da Mata Atlântica (OLIVEIRA FILHO, 2006), entretanto seu caráter de transição desse bioma para o Cerrado, foi critério utilizado para comparação da fauna de vespídeos do presente trabalho. Para essa análise foi utilizado o índice de similaridade de Jaccard, que leva em consideração apenas presença e ausência das espécies. 


\section{RESULTADOS E DISCUSSÃO}

Foram coletados 2239 espécimes de vespas sociais, de 33 espécies e nove gêneros (Tabela 1), apresentando uma maior riqueza de espécies em relação a outros estudos realizados no bioma Cerrado no estado de Minas Gerais, com metodologia de coleta semelhante (ELPINO-CAMPOS et al., 2007; SIMÕES et al., 2012; JACQUES et al., 2015; 2018a). Dessa riqueza, apenas Agelaia angulata é o primeiro registro em Cerrado, até então relatada em floresta ombrófila no Parque Estadual do Rio Doce (SOUZA et al., 2012; SOUZA et al., 2017).

Tabela 1: Número de indivíduos, riqueza de espécies ( $\left.S^{\prime}\right)$, índice de Shannon-Wiener ( $\left.H^{\prime}\right)$ e índice de Berger-Parker (Dpb) das vespas sociais coletadas no Parque Nacional da Serra da Canastra, no município de São Roque de Minas, Minas Gerais, Brasil, durante as quatro campanhas de coletas realizadas, comparando as metodologias de coletas utilizadas.

\begin{tabular}{|c|c|c|c|c|}
\hline & Espécies & Armadilha & Busca ativa & Total \\
\hline 1 & Agelaia angulata (Fabricius, 1804) & 89 & 4 & 93 \\
\hline 2 & Agelaia multipicta (Haliday, 1836) & 57 & 15 & 72 \\
\hline 3 & Agelaia pallipes (Olivier, 1791) & 5 & 2 & 7 \\
\hline 4 & Agelaia vicina (Saussure, 1854) & 24 & 2 & 26 \\
\hline 5 & Apoica gelida Van der Vecht, 1973 & 4 & 1 & 5 \\
\hline 6 & Brachygastra lecheguana (Latreille, 1824) & 3 & 0 & 3 \\
\hline 7 & Mischocyttarus sp. (I) & 0 & 3 & 3 \\
\hline 8 & Mischocyttarus sp. (II) & 0 & 1 & 1 \\
\hline 9 & Mischocyttarus cassununga (R. Von. Ihering, 1903) & 7 & 3 & 10 \\
\hline 10 & Mischocyttarus cerberus (Richards, 1940) & 167 & 1 & 168 \\
\hline 11 & Mischocyttarus drewseni Sausurre, 1856 & 10 & 10 & 20 \\
\hline 12 & Mischocyttarus matogrossensis Zikán, 1935 & 0 & 4 & 4 \\
\hline 13 & Mischocyttarus rotundicolis (Cameron, 1912) & 5 & 25 & 30 \\
\hline 14 & Polistes sp. & 19 & 9 & 28 \\
\hline 15 & Polistes billardieri ruficormis Saussure, 1853 & 63 & 33 & 96 \\
\hline 16 & Polistes cinerascens Saussure, 1854 & 1 & 3 & 4 \\
\hline 17 & Polistes ferreri Saussure, 1853 & 55 & 6 & 61 \\
\hline 18 & Polistes satan Bequaert, 1940 & 40 & 3 & 43 \\
\hline 19 & Polistes simillimus Zikán, 1951 & 49 & 9 & 58 \\
\hline 20 & Polistes subsericius Saussure, 1854 & 14 & 6 & 20 \\
\hline 21 & Polistes versicolor (Olivier, 1971) & 50 & 14 & 64 \\
\hline 22 & Polybia sp. & 1 & 0 & 1 \\
\hline 23 & Polybia chrysothorax (Lechtenstein, 1796) & 2 & 3 & 5 \\
\hline 24 & Polybia fastidiosuscula Saussure, 1854 & 53 & 8 & 61 \\
\hline 25 & Polybia ignobilis (Haliday, 1836) & 98 & 47 & 145 \\
\hline 26 & Polybia jurinei Saussure, 1854 & 37 & 6 & 43 \\
\hline 27 & Polybia occidentalis (Olivier, 1971) & 139 & 24 & 163 \\
\hline 28 & Polybia paulista (R. Von. Ihering, 1896) & 356 & 19 & 375 \\
\hline 29 & Polybia quadricincta Saussure, 1854 & 1 & 0 & 1 \\
\hline 30 & Polybia sericea (Olivier, 1971) & 534 & 91 & 625 \\
\hline 31 & Protonectarina sylveirae (Saussure, 1854) & 0 & 2 & 2 \\
\hline 32 & Protopolybia sedula (Saussure, 1854) & 0 & 1 & 1 \\
\hline \multirow[t]{5}{*}{33} & Pseudopolybia vespiceps (Saussure, 1864) & 0 & 1 & 1 \\
\hline & Total de Indivíduos & 1883 & 356 & 2239 \\
\hline & Riqueza de espécies (S’) & 27 & 30 & 33 \\
\hline & Índice de Shannon-Wiener (H`) & 2,412 & 2,672 & 2,522 \\
\hline & Índice de Berger-Parker (Dpb) & 0,2836 & 0,2556 & 0,2791 \\
\hline
\end{tabular}

Comparando os trabalhos realizados no Cerrado de Minas Gerais, o presente estudo apresenta mais semelhança com o estudo realizado por Jacques et al. (2015; 2018a) (Figura 2). Isso pode ser explicado devido à proximidade $(70 \mathrm{~km}$ ) e semelhança da fitofisionomia desses estudos (SOUZA et al, 2015). O estudo realizado por Brunnisman et al. (2016), por ser uma área de transição de Mata Atlântica e Cerrado apresentou a menor similaridade entre os trabalhos analisados, como esperado. 


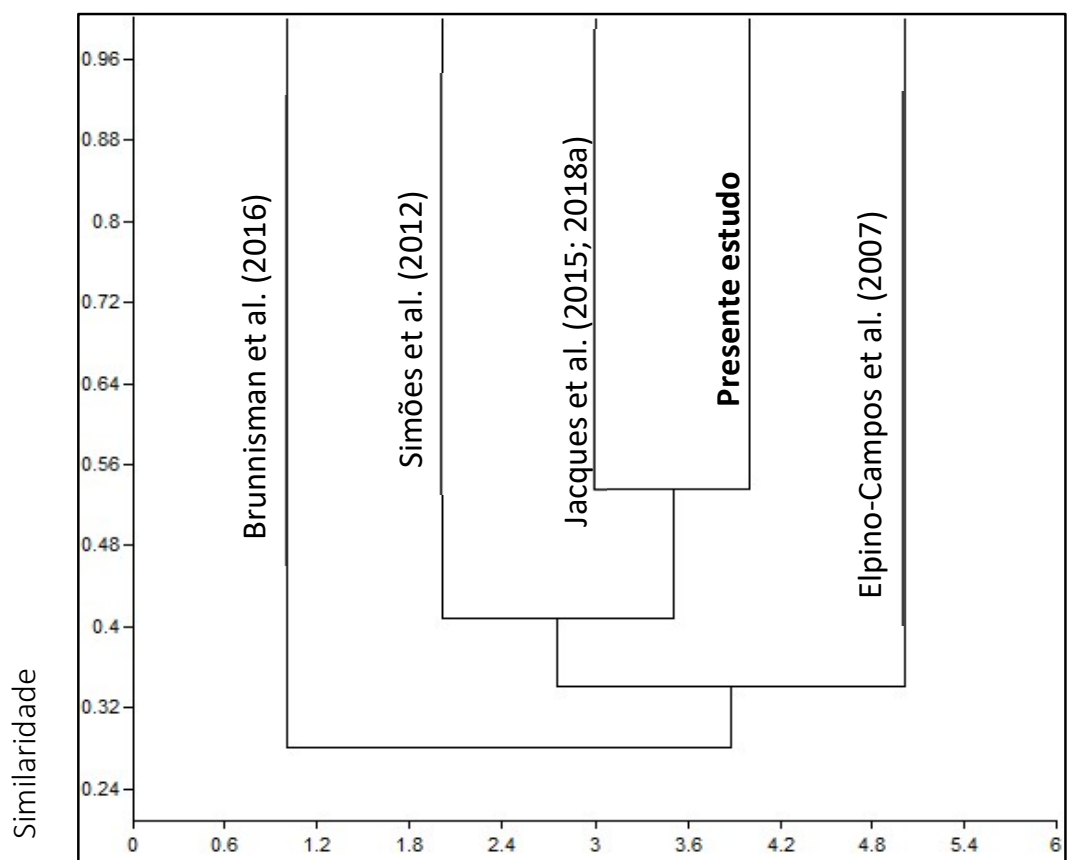

Figura 2: Análise de similaridade (Cluster analysis) entre os trabalhos de diversidade de vespas sociais realizados no bioma Cerrado, no estado de Minas Gerais.

Obteve-se um baixo índice de dominância $(\mathrm{Dpb}=0,27)$, sendo as espécies mais abundantes: Polybia sericea (Olivier, 1971), com 625 indivíduos coletados; Polybia paulista (R. Von. Ihering, 1896), com 375 indivíduos coletados e Mischocyttarus cerberus (Richards, 1940), com 168 indivíduos coletados. Esse resultado por ser explicado por diferentes condições, primeiro, essas espécies têm ampla distribuição geográfica em todas as regiões (RICHARDS, 1978); segundo, ocorrem em diferentes fitofisionomias de biomas como Cerrado (DINIZ et al., 1994), Mata Atlântica (SOUZA et al., 2017); além de $P$. paulista e $M$. cerberus serem consideradas sinantrópicas (OLIVEIRA et al., 2017).

A área 1 - Cachoeira do Rolinho obteve alto número de indivíduos coletados, totalizando 1078 vespas sociais coletadas, sendo que 962 foram coletadas por armadilhas atrativas e 116 por busca ativa. Apesar desse alto número de indivíduos coletados, esta área foi a que obteve menor riqueza de espécies $\left(S^{\prime}=17\right)$, isso se deve ao fato da alta dominância de poucas espécies, apresentando o maior índice de dominância (Dpb $=0.4406$ ) dentre as três áreas de estudo (Tabela 2).

Tabela 2: Número de indivíduos, riqueza de espécies ( $\left.\mathrm{S}^{\prime}\right)$, índice de Shannon-Wiener ( $\mathrm{H}^{\prime}$ ) e índice de Berger-Parker (Dpb) das vespas sociais coletadas no Parque Nacional da Serra da Canastra, no município de São Roque de Minas, Minas Gerais, Brasil, comparando a quantidade de vespas sociais coletadas em cada área de estudo, no total das quatro campanhas.

\begin{tabular}{|c|c|c|c|c|c|c|c|c|c|c|}
\hline & \multirow[b]{2}{*}{ Espécies } & \multicolumn{3}{|c|}{$\begin{array}{l}\text { Área } 1 \text { - Cachoeira do } \\
\text { Rolinho }\end{array}$} & \multicolumn{3}{|c|}{$\begin{array}{l}\text { Área } 2 \text { - Cachoeira Casca } \\
\text { d'Anta }\end{array}$} & \multicolumn{3}{|c|}{ Área 3 Parte Baixa } \\
\hline & & $\begin{array}{l}\text { Armadi } \\
\text { Iha }\end{array}$ & $\begin{array}{l}\text { Busca } \\
\text { Ativa }\end{array}$ & Total & $\begin{array}{l}\text { Armadi } \\
\text { lha }\end{array}$ & $\begin{array}{l}\text { Busca } \\
\text { Ativa }\end{array}$ & Total & $\begin{array}{l}\text { Armadi } \\
\text { lha }\end{array}$ & $\begin{array}{l}\text { Busca } \\
\text { Ativa }\end{array}$ & Total \\
\hline 1 & Agelaia angulata (Fabricius, 1804) & 0 & 0 & 0 & 5 & 0 & 5 & 84 & 4 & 88 \\
\hline 2 & Agelaia multipicta (Haliday, 1836) & 1 & 0 & 1 & 0 & 0 & 0 & 56 & 15 & 71 \\
\hline 3 & Agelaia pallipes (Olivier, 1791) & 0 & 0 & 0 & 0 & 0 & 0 & 5 & 2 & 7 \\
\hline 4 & Agelaia vicina (Saussure, 1854) & 0 & 0 & 0 & 0 & 0 & 0 & 24 & 2 & 26 \\
\hline 5 & $\begin{array}{l}\text { Apoica gelida Van der Vecht, } 1973 \\
\text { Brachygastra lecheguana (Latreille, }\end{array}$ & 1 & 1 & 2 & 1 & 0 & 1 & 2 & 0 & 2 \\
\hline 6 & 1824) & 2 & 0 & 2 & 1 & 0 & 1 & 0 & 0 & 0 \\
\hline 7 & Mischocyttarus sp. (I) & 0 & 0 & 0 & 0 & 0 & 0 & 0 & 3 & 3 \\
\hline 8 & Mischocyttarus sp. (II) & 0 & 0 & 0 & 0 & 0 & 0 & 0 & 1 & 1 \\
\hline 9 & Mischocyttarus cassununga (R. Von. & 5 & 0 & 5 & 0 & 0 & 0 & 2 & 3 & 5 \\
\hline
\end{tabular}




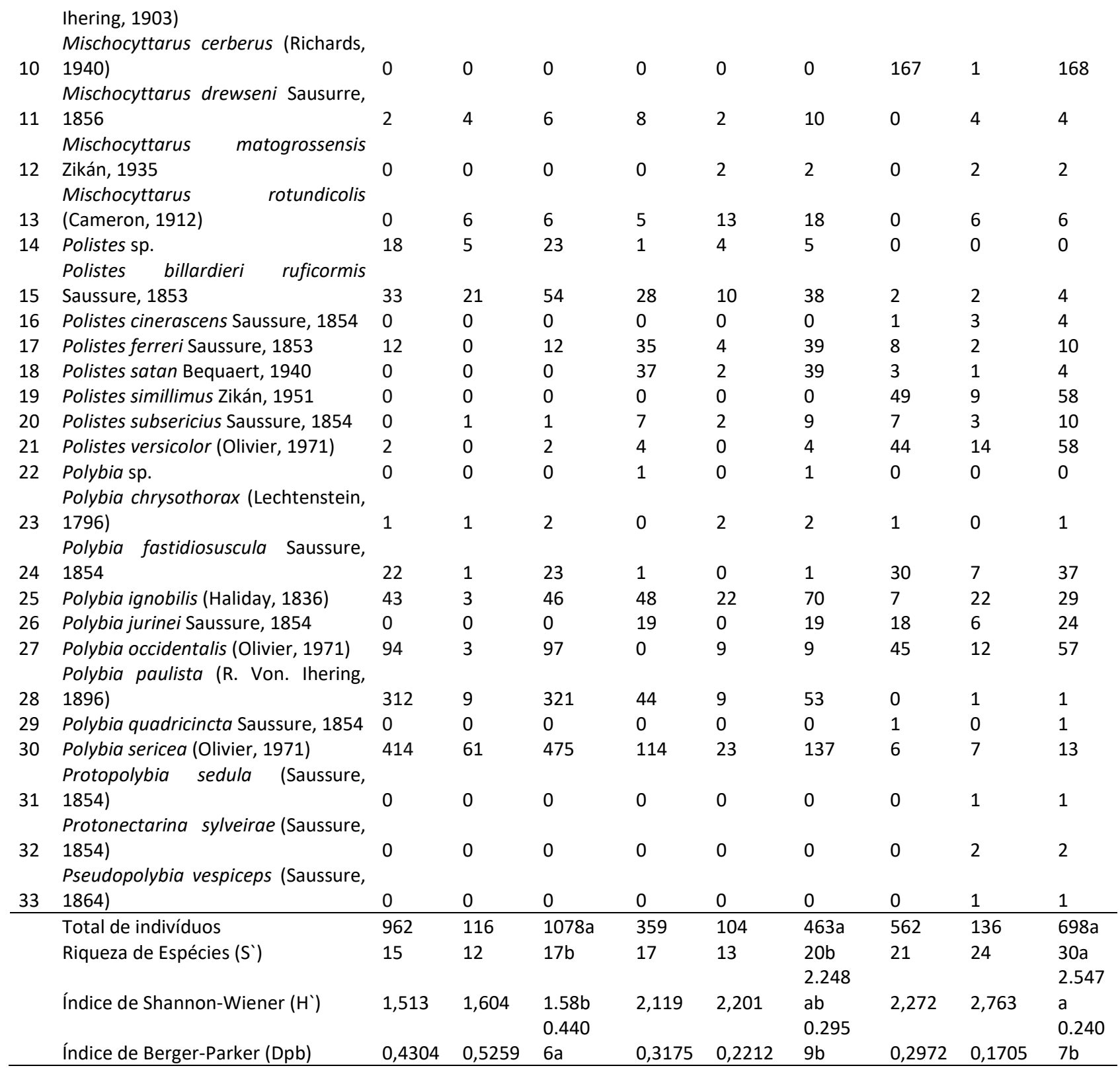

A Área 3 - Parte Baixa apresentou a maior riqueza de espécies e índice de diversidade. Isto pode ter ocorrido pois esta área apresenta um ambiente muito diversificado, pois é uma região de transição entre Mata Atlântica e Cerrado, além de conter em alguns pontos, influência antrópica, como quiosques, banheiros, postes de iluminação e trilhas. Isto pode ajudar a explicar o grande número de espécies coletadas, pois ambientes estruturalmente mais heterogêneos e complexos podem favorecer a coexistência de um maior número de espécies, devido à maior oferta de microhabitats, maior proteção contra predadores e maior disponibilidade e diversidade de recursos alimentares e de substratos para nidificação (SANTOS et al., 2007; SOUZA et al., 2012).

M. cerberus foi a espécie mais abundante nessa área, totalizando 168 indivíduos coletados. Observase também uma alta frequência de coleta das espécies Polistes simillimus Zikán, 1951 e Polistes versicolor (Olivier, 1971). Essa condição pode ser explicada, pois essas espécies são sinantrópicas e nidificam tanto em edificações urbanas como em ambientes naturais (OLIVEIRA et al., 2017), o que facilita sua localização.

As espécies Agelaia angulata (Fabricius, 1804), Agelaia pallipes (Olivier, 1791), Agelaia vicina 
(Saussure, 1854), Mischocyttarus sp. (I), Mischocyttarus sp. (II), Mischocyttarus cerberus (Richards, 1940), Protopolybia sedula (Saussure, 1854) e Protonectarina sylveirae (Saussure, 1854) foram coletadas exclusivamente nessa área. Há espécies que são mais generalistas (eurióicas), e outras mais restritas quanto ao tipo de recurso explorado (estenóicas) (CRUZ et al., 2006, SOUZA et al., 2014a). Diferentes fatores podem influenciar a distribuição dessas vespas sociais, como disponibilidade de água, alimento e distintos fatores abióticos (SANTOS et al., 1998; CRUZ et al., 2006); substrato para nidificação, sejam naturais, como cavidades em troncos, termiteiros, barrancos, face abaxial de folhas coriáceas, e construções humanas (OLIVEIRA et al., 2017; FRANCISCO et al., 2018); e estrutura do ambiente, como grau de conservação e/ou de regeneração (SOUZA et al., 2010). Entretanto, para confirmar a exclusividade de uma espécie em um ambiente, o dado mais confiável seja o registro das colônias (SOUZA et al., 2010), pois uma espécie pode nidificar em um ambiente e explorar recursos em outros (DINIZ et al., 1994; SILVA-PEREIRA et al., 2006).

Algumas espécies de vespas sociais podem viabilizar informações sobre a situação ambiental de determinadas áreas, através de sua presença e/ou ausência, agindo assim como bioindicadores de qualidade ambiental (SOUZA et al., 2010). A presença conjunta das espécies Polybia fastidiosuscula Saussure, 1854 e Pseudopolybia vespiceps (Saussure, 1864) na área 3 sugerem um bom estado de conservação dessa área (SOUZA et al., 2010).

Através da análise de similaridade (Cluster analysis) (Figura 3), observou-se que a Área 1 - Cachoeira Rolinho e a Área 2 - Cachoeira Casca d'Anta são mais similares quando comparada a fauna das vespas sociais, apresentando um índice de similaridade acima de $40 \%$. Fica notório a heterogeneidade da Área 3 - Parte Baixa, em relação à área 1 e 2, sendo que ela apresenta uma similaridade abaixo de $20 \%$ com as demais áreas. Isso se deve ao fato das áreas de estudos 1 e 2 estarem inseridas no mesmo tipo de fitofisionomia, que é o Cerrado stricto sensu, enquanto a área 3 está inserida em um fragmento da fitofisionomia de Floresta Estacional Semidecidual.

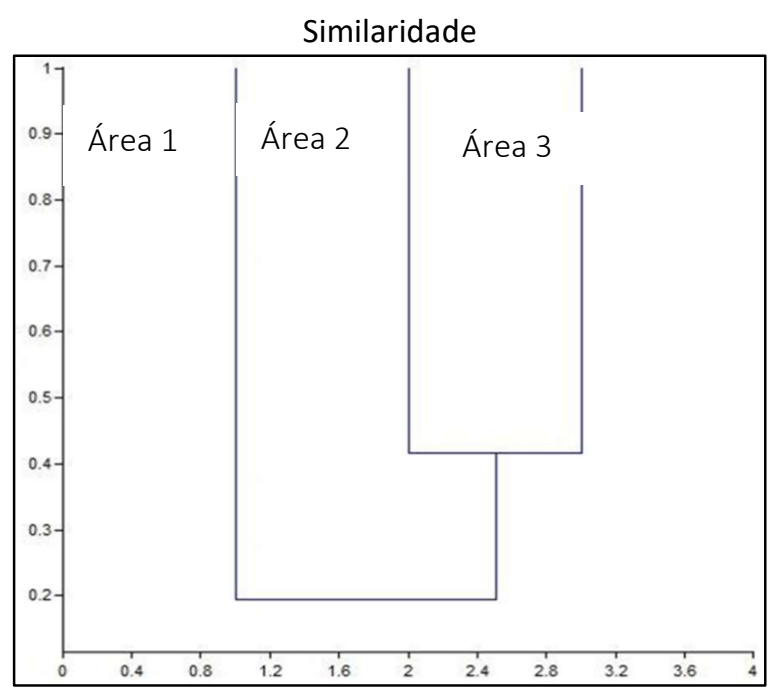

Figura 3: Análise de similaridade (Cluster analysis) entre as faunas de vespas sociais coletadas nas três áreas de estudo, levando em consideração a ocorrência e a abundância das espécies em cada área.

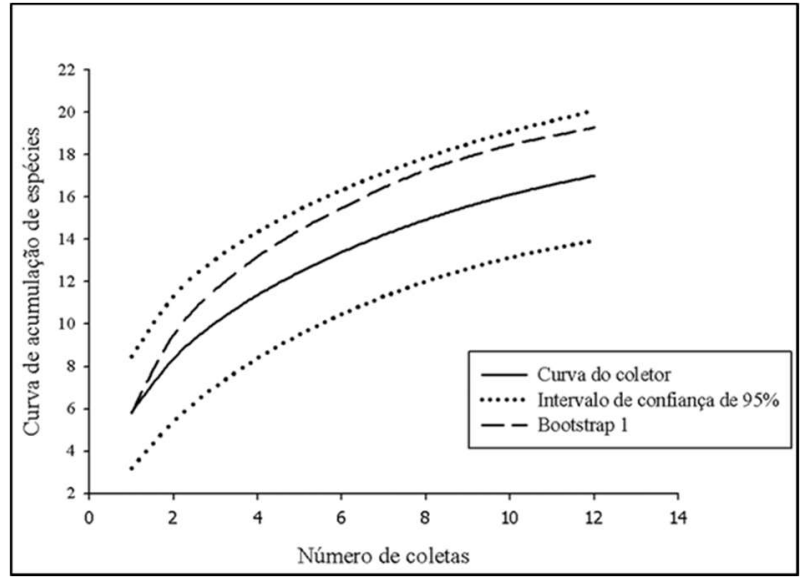

Figura 4: Curva de acumulação de espécies da Área 1 Cachoeira do Rolinho, com intervalo de confiança de 95\%, utilizando o estimador Bootstrap 1.

Levando em consideração a distância entre as áreas de estudo, nota-se que a Área 2 é mais próxima 
da Área 3, tendo uma distância aproximada em linha reta, de 2 km, em comparação com a distância entre a Área 1 e a Área 2, que apresenta uma distância aproximada de $12 \mathrm{~km}$ em linha reta. Com isso fica nítido que a similaridade das fitofisionomias é mais relevante que a distâncias entre as áreas de estudo.

A curva de acumulação do coletor das três áreas (Figuras 4, 5 e 6) ficou dentro do intervalo de confiança de 95\%, sendo equivalente ao estimador Bootstrap 1, mostrando um esforço amostral suficiente. A curva do coletor das três áreas conseguiu atingir a assíntota, nos indicando que $95 \%$ da riqueza total das áreas de estudo foram amostradas (SCHILLING et al., 2008).

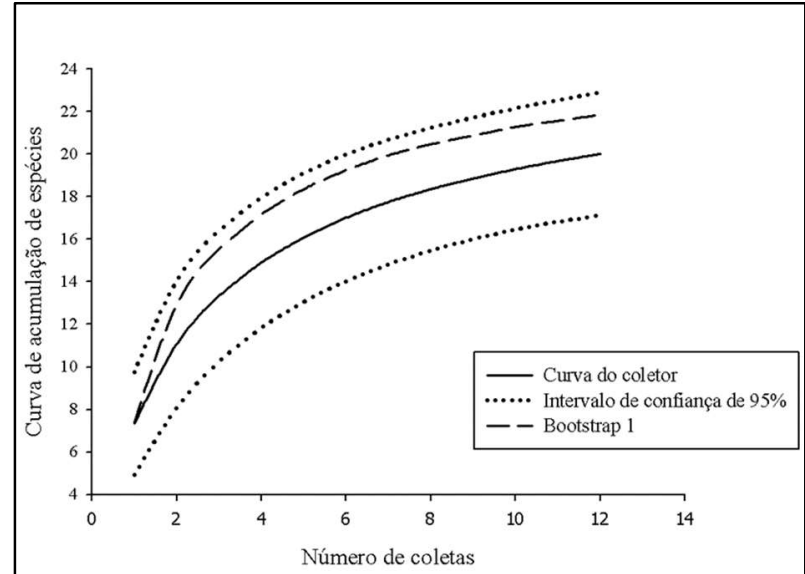

Figura 5: Curva de acumulação de espécies da Área 2Cachoeira Casca d'Anta, com intervalo de confiança de 95\%, utilizando o estimador Bootstrap 1.

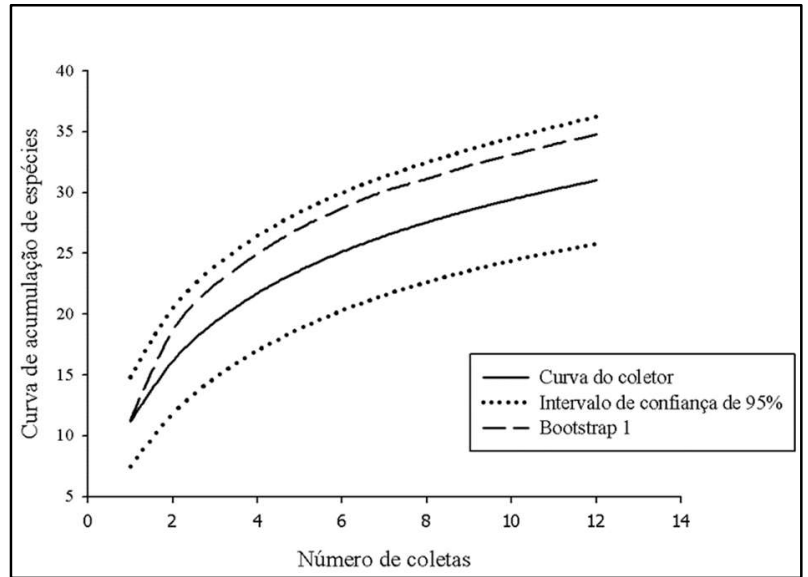

Figura 6: Curva de acumulação de espécies da Área 3 Parte Baixa, com intervalo de confiança de 95\%, utilizando o estimador Bootstrap 1.

\section{CONCLUSÕES}

Este estudo, que é o primeiro levantamento de vespas sociais na Serra da Canastra, encontrou uma alta riqueza dessas vespas, comparando com outras áreas de Cerrado, mostrando ser um ambiente rico em biodiversidade e ainda pouco explorado. Além disso, fica claro que a similaridade das fitofisionomias é mais relevante na composição de espécies que a distâncias entre as áreas de estudo.

\section{REFERÊNCIAS}

ANDENA, R. B.; CARPENTER, J. M.; PICKETT, K. M.. Phylogenetic analysis of species of the Neotropical social wasp Epipona Latreille, 1802 (Hymenoptera, Vespidae, Polistinae, Epiponini). ZooKeys, v.20, p.385-398, 2009. DOI: http://doi.org/10.3897/zookeys.20.79

BARBOSA, B. C.; DETONI, M.; MACIEL, T. T.; PREZOTO, F.. Studies of social wasp diversity in Brazil: Over 30 years of research, advancements and priorities. Sociobiology, v.63, n.3, p.858-880, 2016.

BRUNISMANN, A. G.; SOUZA, M. M.; PIRES, E. P.; COELHO, E. L.; MILANI, L. R.. Social wasps (Hymenoptera: Vespidae) in Deciduous Seasonal Forest in Southeastern Brazil. Journal of Entomology and Zoology Studies, v.4, p.447-452, 2016.

BUENO, E. T.; SOUZA, M. M.; CLEMENTE, M. A.. The Effect of Forest Fragmentation on Polistinae. Sociobiology, v.66, p.508-514, 2019. DOI:

http://dx.doi.org/10.13102/sociobiology.v66i3.4378
CARPENTER, J. M.. Synonymy of the genus Marimbonda Richards 1978, with Leipomeles Mobius, 1856 (Hymenoptera: Vespidae; Polistinae), and a new key to the genera of paper wasps of the New World. American Museum Novitates, v.3465, p.1-16, 2004. DOI: http://doi.org/10.1206/00030082(2004)465<0001:SOTGMR>2.0.CO;2

CLEMENTE, M. A.. Vespas Sociais (Hymenoptera, Vespidae) do Parque Estadual do Ibitipoca/MG: estrutura, composição e visitação floral. Dissertação (Mestrado) - Universidade Federal de Juiz de Fora, Juiz de Fora, 2009.

COWELL, R. K.. EstimateS: Statistical estimation of species richness and shared species from samples. (Software and User's Guide), Version 9 and earlier. 2013.

CRUZ, J. D.; GIANNOTTI, E.; SANTOS, G. M. M.; BICHARA FILHO, C. C.; ROCHA, A. A.. Nest site selection and flying capa-city of netropical wasp Angiopolybia pallens 
(Hymenoptera: Vespidae) in the Atlantic Rain Forest, Bahia State, Brazil. Sociobiology, v.47, p.739-749, 2006.

DINIZ, I. R.; KITAYAMA, K.. Colony densities and preferences for nest habitats of some social wasps in Mato Grosso State, Brasil (Hymenoptera: Vespidae). Journal of Hymenoptera Research, v.3, n.1, p.133-143, 1994.

DRUMMOND, G. M.; MARTINS, C. S.; MACHADO, A. B. M.; SEBAIO, F. A.; ANTONINI, Y.. Biodiversidade em Minas Gerais: um atlas para a conservação. Belo Horizonte: Fundação Biodiversitas, 2005.

EITEN, G.. Vegetação do Cerrado. In: PINTO, M. N.. Cerrado: caracterização, ocupação e perspectivas. Brasília: UnB/SEMATEC, 1994. p.17-73.

ELPINO-CAMPOS, A.; DEL-CLARO, K.; PREZOTO, F.. Diversity of social wasps (Hymenoptera: Vespidae) in Cerrado fragments of Uberlândia, Minas Gerais State, Brazil. Neotropical Entomology, v.36, p.685-692, 2007.

FELFILI, J. M.; SILVA JÚNIOR, M. C.; REZENDE, A. V.; NOGUEIRA, P. E.; WALTER, B. M. T.; SILVA, M. A.; ENCINAS, J. I.. Comparação florística e fitossociológica do Cerrado nas Chapadas Pratinha e dos Veadeiros. In: LEITE, L.; SAITO, C. H.. Contribuição ao conhecimento ecológico do cerrado. Brasília: Universidade de Brasília, 1997. p.6-11.

FELFILI, J. M.; SILVA JÚNIOR, M. C.. Biogeografia do Bioma Cerrado: estudo fitofisionômico da Chapada do Espigão Mestre do São Francisco. 2001.

FRANCISCO, G. S.; SOUZA, M. M.; CLEMENTE, M.; BRUNISMANN, A. G.. Substrato vegetal utilizado para nidificação de vespas sociais (Hymenoptera, Vespidae) em Floresta Decidual. Revista Agrogeoambiental, v.10, n.3, p.35-45. 2018. DOI: http://dx.doi.org/10.18406/23161817v10n320181162

GRAÇA, M. B.; SOMAVILLA, A.. Effects of forest fragmentation on community patterns of social wasps (Hymenoptera: Vespidae) in Central Amazon. Austral Entomology, v.58, p.657-665, 2018. DOI: https://doi.org/10.1111/aen.12380

HAMMER, O.; HARPER, D. A. T.; RYAN, P. D.. Past: paleontological statistics software package for education and data analysis. Palaeontologica Electronica, v.4, p.1-9, 2005.

HERMES, M. G; KÖHLER, A.. The flower-visiting social wasps (Hymenoptera, Vespidae, Polistinae) in two areas of Rio Grande do Sul State, Southern Brazil. Revista Brasileira de Entomologia, v.50, p.268-274, 2006. DOI: http://doi.org/10.1590/S0085-56262006000200008

HERMES, M. G.; SOMAVILLA, A.; ANDENA, S. R.. Vespidae in catálogo taxonômico da fauna do Brasil. PNUD, 2020.

IBGE. Instituto Brasileiro de Geografia e Estatística. Mapas de Biomas e de Vegetação. Brasília: IBGE, 2004.

JACQUES, G. C.; SOUZA, M. M.; COELHO, H. J.; VICENTE, L. O.; SILVEIRA, L. C. P.. Diversity of Social Wasps (Hymenoptera: Vespidae: Polistinae) in an Agricultural Environment in Bambuí, Minas Gerais, Brazil. Sociobiology, v.62, p.439-445, 2015. DOI:

http://doi.org/10.13102/sociobiology.v62i3.738

JACQUES, G. C.; PIKART, T. G.; SANTOS, V. S.; VICENTE, L. O. SILVEIRA, L. C. P.. Niche overlap and daily activity pattern of social wasps (Vespidae: Polistinae) in kale crops. Sociobiology, v.65, p.312-319, 2018a. DOI: http://foi.org/10.13102/sociobiology.v65i2.2670

JACQUES, G. C.; PIRES, E. P.; HERMES, M. G.; FARIA, L. D. B.; SOUZA, M. M.; SILVEIRA, L. C. P.. Evaluating the efficiency of different sampling methods to survey social wasps (Vespidae: Polistinae) in an anthropized environment. Sociobiology, v.65, n.3, p.515-523, 2018b. DOI: http://doi.org/10.13102/sociobiology.v65i3.2849

JACQUES, G. C.; OLIVEIRA, D. C.; SOUZA, M. M.; SILVEIRA, L. C. P.. The use of Polistes versicolor (Olivier, 1971) in the control of Ascia monuste orseis (Godart, 1819) in kale cultivation. Agrogeoambiental, v.11, p.96-106, 2019.

KLINK, C. A.; MACHADO, R. B.. A conservação do Cerrado brasileiro. Megadiversidade, v.1, p.147-155, 2005.

MACHADO, R. B.; RAMOS NETO, M. B.; PEREIRA, P.; CALDAS E.; GONÇALVES, D.; SANTOS, N.; TABOR, K.; STEININGER, M.. Estimativas de perda da área do Cerrado brasileiro. Brasília: Conservation International do Brasil, 2004.

MACIEL, T. T.. Coleta de Vespas Sociais em Estudos de Diversidade: Estado da Arte e Otimização do Método de Armadilhas Atrativas. Dissertação (Mestrado em Ciências Biológicas: Comportamento Animal) - Universidade Federal de Juiz de Fora, Juiz de Fora, 2017.

MYERS, N.; MITTERMEIER, R. A.; MITTERMEIER, C. G.; FONSECA, G. A. B.; KENT, J.. Biodiversity hotspots for conservation priorities. Nature, v.403, p.853-858, 2000.

OLIVEIRA FILHO, A. T.; JARENKOW, J. A.; RODAL, M. J. N. Floristic relationships of seasonally dry forests of eastern South America based on tree species distribution patterns. In: PENNINGTON, R. T.; LEWIS, G. P.; RATTER, J.. Neotropical savannas and seasonally dry forests: plant diversity, biogeography, and conservation. Oxford: Taylor Francis CRC Press, 2006. p.59-192.

OLIVEIRA, T. C. T.; SOUZA, M. M.; PIRES, E. P.. Nesting habits of social wasps (Hymenoptera: Vespidae) in forest fragments associated with anthropic areas in southeastern Brazil. Sociobiology, v.36, p.189-196, 2017. DOI: http://dx.doi.org/10.13102/sociobiology.v64i1.1073

PREZOTO, F.; MACHADO, V. L. L.. Ação de Polistes (Aphanilopterus) simillimus Zikán (Hymenoptera, Vespidae) no controle de Spodoptera frugiperda (Smith) (Lepidoptera, Noctuidae). Revista Brasileira de Zoologia, v.16, p.841-850, 1999.

PREZOTO, F.; CORTES, S. A. O.; MELO, A. C.. Vespas: de vilãs a parceiras. Ciência Hoje, v.48, p.70-73, 2008.

PREZOTO, F.; MACIEL, T. T.; DETONI, M.; MAYORQUIN, A. Z.; BARBOSA, B. C.. Pest Control Potential of Social Wasps in Small Farms and Urban Gardens. Insects, v.10, p.192, 2019. DOI: $\underline{\text { http://doi.org/10.3390/insects10070192 }}$ 
RATTER, J.; BRIDGEWATER, S.; RIBEIRO, J. F.. Analysis of the floristic composition of the Brazilian Cerrado vegetation. III: comparison of the woody vegetation of 376 areas.

Edinburgh Journal of Botany, v.60, p.57-109, 2003.

RICHARDS, O. W.. The social wasps of the America, excluding the Vespinae. London: British Museum (Natural History), 1978.

SANTOS, G. M. M.; GOBBI, N.. Nesting habits and colonial productivity of Polistes canadensis canadensis (L.) (Hymenoptera: Vespidae) in a caatinga area, Bahia StateBrazil. Journal of Advanced Zoology, v.19, p.63-69, 1998.

SANTOS, A. J.. Estimativas de riqueza de espécies. In: RUDRAN, R.; CULLEN, L.; VALLADARES-PADUA, C.. Métodos de estudo em biologia da conservação e manejo da vida terrestre. Curitiba: Universidade Federal do Paraná, 2003. p.19-41.

SANTOS, G. M. M.; FILHO, C. C. B.; RESENDE, J. J.; CRUZ, J. D.; MARQUES, O. M.. Diversity and community structures of social wasps (Hymenoptera: Vespidae) in three ecosystems in Itaparica Island, Bahia State, Brazil. Neotropical Entomology, v.36, p.180-185, 2007.

SCHILLING, A. C.; BATISTA, J. L. F.. Curva de acumulação de espécies e suficiência amostral em florestas tropicais. Brazilian Journal of Botany, v.31, n.1, p.179-187, 2008.

SIMÕES, M. H.; CUOZZO, M. D.; FRIERO-COSTA, F. A.. Diversity of social wasps (Hymenoptera, Vespidae) in Cerrado biome of the southern of the state of Minas Gerais, Brazil. Iheringia Série Zoologia, v.102, p.292-297, 2012. DOI: http://doi.org/10.15 90/S0073-47212012000300007

SILVA, J. M. C.; BATES, J. M.. Biogeographic patterns and conservation in the South American Cerrado: a tropical savanna hotspot. BioScience, v.52, p.225-233, 2002.

SILVA-PEREIRA, V.; SANTOS, G. M. M.. Diversity in bee (Hymenopetra, Apoidea) and social wasps (Hymenoptera, Vespidae) comumnity in campos rupestres, Bahia, Brazil.
Neotropical Entomology, v.35, p.165-174, 2006. DOI: http://doi.org/10.1590/S15 19-566X2006000200003

SOUZA, M. M.; PREZOTO, F.. Diversity of social wasps (Hymenoptera, Vespidae) in Semideciduous Forest and Cerrado (Savanna) regions in Brazil. Sociobiology, v.47, p.135-147, 2006.

SOUZA, M. M.; LOUZADA, J.; SERRÃO, J. E.; ZANUNCIO, J. C. Social wasps (Hymenoptera: Vespidae) as indicators of conservation degree of riparian forests in Southeast Brazil. Sociobiology, v.56, p.387-396, 2010.

SOUZA, M. M.; PIRES, E. P.; FERREIRA, M.; LADEIRA, T. E.; PEREIRA, M.; ELPINO-CAMPOS, A.; ZANUNCIO, J. C.. Biodiversidade de vespas sociais (Hymenoptera: Vespidae) do Parque Estadual do Rio Doce, Minas Gerais, Brasil. Sociobiology, v.5, p.4-19, 2012.

SOUZA, M. M.; PIRES, E. P.; PREZOTO, F.. Seasonal richness and composition of social wasps (Hymenoptera, Vespidae) in areas of Cerrado biome in Barroso, Minas Gearis State, Brazil. Bioscience Journal, v.30, p.539-545, 2014a.

SOUZA, M. M; PERILLO, L. N.; BARBOSA, B. C.; PREZOTO, F. Use of flight interception traps of Malaise type and Attractive Traps for social wasps record (Vespidae: Polistinae). Sociobiology, v.62, p.450, 2015.

SOUZA, M. M.; BRUNISMANN, A. G.; CLEMENTE, M. A.. Species composition, relative abundance and distribution of social wasps fauna on different ecosystems. Sociobiology, v.64, n.4, p.456-465, 2017. DOI: http://doi.org/10.13102/sociobiology.v64i4.1839

SOUZA, M. M.; GUEDES, G. T.; BUENO, E. T.; MILANI, L. R.; SOUZA, A. S. B.. Social Wasps (Hymenoptera, Polistinae) from the Brazilian Savanna. Sociobiology, v.67, p.129-138, 2020a. DOI:

http://doi.org/10.13102/sociobiology.v67i2.4958

SOUZA, M. M.; GUEDES, G. T.; MILANI, L. R.; SOUZA, A. S. B.; GOMES, P. P.. Social Wasps (Vespidae: Polistinae) from the Brazilian Atlantic Forest. Sociobiology, v.67, p.01-12, 2020b.

A CBPC - Companhia Brasileira de Produção Científica (CNPJ: 11.221.422/0001-03) detém os direitos materiais desta publicação. Os direitos referem-se à publicação do trabalho em qualquer parte do mundo, incluindo os direitos às renovações, expansões e disseminações da contribuição, bem como outros direitos subsidiários. Todos os trabalhos publicados eletronicamente poderão posteriormente ser publicados em coletâneas impressas sob coordenação da Sustenere Publishing, da Companhia Brasileira de Produção Científica e seus parceiros autorizados. Os (as) autores (as) preservam os direitos autorais, mas não têm permissão para a publicação da contribuição em outro meio, impresso ou digital, em português ou em tradução. 